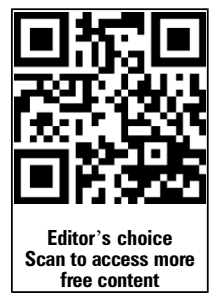

\title{
An introduction to quality improvement in paediatrics and child health
}

\author{
C Lemer, ${ }^{1}$ C R L H Cheung, ${ }^{2}$ R E Klaber ${ }^{3}$
}

\begin{abstract}
${ }^{1}$ Evelina Children's Hospital, Guy's and St Thomas' NHS Foundation Trust, London, UK ${ }^{2}$ Department of Paediatrics, Croydon University Hospital London, UK

${ }^{3}$ Department of Paediatrics, Imperial College Healthcare NHS Trust, London, UK
\end{abstract}

\section{Correspondence to}

Dr Bob Klaber, Department of Paediatrics, Imperial College Healthcare NHS Trust, St Mary's Hospital, 6-10 The Bays Building, Off South Wharf Road London W2 1NY, UK; Robert.klaber@imperial.nhs.uk

Received 17 June 2013

Accepted 3 July 2013

Published Online First

1 August 2013
To cite: Lemer $C$ Cheung CRLH, Klaber RE. Arch Dis Child Educ Pract Ed 2013;98:175-180.

\begin{abstract}
Quality improvement is a focus on changing the way in which patient care is delivered. Central to this is developing a more rounded, patientfocused approach to the design and delivery of care. Here, in the first of a series, we introduce the concepts of quality improvement, explain why quality improvement should matter to

paediatricians and give some pointers as to where and how paediatricians can learn and do more.
\end{abstract}

\section{WHAT IS QUALITY IMPROVEMENT- AN EXAMPLE}

Imagine you are in a very long hospital corridor. This corridor is so long, in fact, that notes are pedalled along it using foot scooters. You stop and ask the staff what they do in the hospital. Their response surprises you: "We have two jobs: doing our job and improving it."

It is perhaps unlikely that this would be the reaction of employees in most hospitals across the world; the more likely response would be a tribal one: "I'm a doctor" or "I'm a nurse." But that really is the response that you get at Jönköping, Sweden. Jönköping is a hospital that serves a small town in Sweden and is about the same size as a UK District General Hospital. Jönköping has spent over a decade focusing relentlessly on the quality of care that is delivered, and, most importantly, on changing the culture of the hospital.

One example of this focus on quality is the amazing story of the evolution of haemodialysis. When a young engineer required haemodialysis he asked of his nurse, "Why can't I learn to do what you are doing to manage my haemodialysis?" The response from the nurse was extraordinary-she agreed with him. Over the next few months the two went on a journey, which was supported by the hospital. The nurse changed from caregiver to coach and succeeded in training the young engineer to manage his own haemodialysis. As the engineer became more and more capable and increasingly independent, other patients started to notice. So, the engineer and the nurse started to train others. Now, a few years later, over $50 \%$ of the patients have haemodialysis self-manage: from young adults to retirees, they all manage their own long-term condition with support from healthcare professionals. Patients are supplied with swipe card access to the unit so that they can dialyse at times that suit them and, in response to patient feedback, exercise equipment has been installed so that the patients can cycle on an exercise bike while their dialysis takes place. In fact, Jönköping has gone even further: the nurses now help the patients to rewrite their curriculum vitaes, including their new clinical skills, so that they can benefit professionally from their new experience, beyond just their clinical care.

\section{WHAT IS QUALITY IMPROVEMENT- A DEFINITION}

Although hugely innovative, the hospital in Jönköping is not alone; there are many healthcare organisations that have embraced focusing on clinical care in this way. This focus on changing how care is delivered has been called quality improvement, which is often shortened to QI. Underpinning this is a focus on quality that encapsulates the care that patients receive in a rounded, patientfocused manner. Many definitions of quality in healthcare exist, but perhaps the best known comes from the Institute of Medicine, which identified six domains of quality. ${ }^{1}$ These are illustrated in figure 1. 


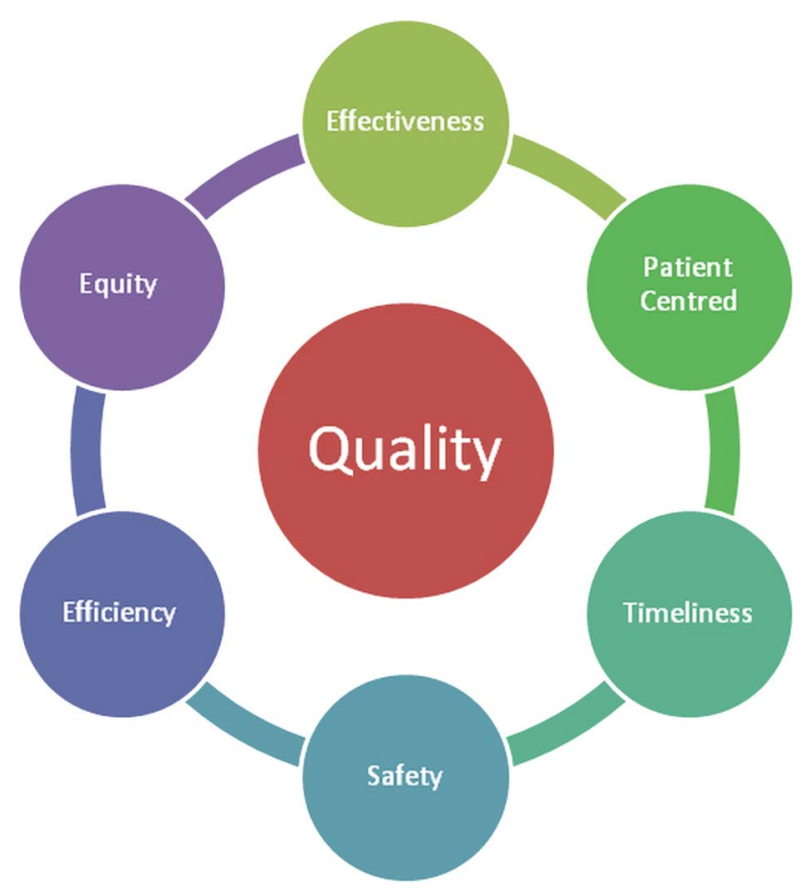

Figure 1 Institute of Medicine's six domains of quality.

\section{WHY SHOULD QUALITY AND QUALITY IMPROVEMENT MATTER TO PAEDIATRICIANS?}

Clinicians choose to become paediatricians for a variety of reasons. For many, part of the attraction is the ability to focus on the whole child or young person within the context of the whole family. Our training and conversations with patients and their families impart us with an understanding of the role that community and societal factors play in the health of our patients. This broader focus often leads child health professionals to think beyond the patient they see in a ward bed or in a clinic consultation. The recent interest generated by the Royal College of Paediatrics and Child Health (RCPCH) meeting on improving and changing service provision-'Child Health in the UK' - and the extensive discussions around 'Facing the Future' show that paediatricians are proactively engaging with important issues about how the delivery of care now and in future. ${ }^{2} 3$

Knowledge of how to change and improve individual services is not yet widespread, and few paediatricians are formally trained in quality improvement methodologies. However this is changing-through support from institutions such as the $\mathrm{RCPCH}$, the Institute for Healthcare Improvement and the now defunct National Institute for Innovation and Improvement-on the back of hospital-led initiatives like EQUIP at Great Ormond Street Hospital in London ${ }^{4}$ and through the energy and commitment of increasing numbers of dedicated and motivated individuals.

As the RCPCH looks to review the future delivery models of healthcare for children and young people, it is very timely for paediatricians in the UK and beyond to start to ask themselves what their role should entail in future. Is it enough to just treat the patient in front of us? Or is it our professional responsibility to improve the services we work in too? If it is both, the launch of RCPCH Quality Improvement Training Programme provides a real opportunity for paediatrics to be the first specialty to embrace quality improvement as a core part of our professional responsibilities and to provide a new generation with the right skills to turn this potential into reality.

This introductory paper explains the domains of quality, contextualising them through a number of examples of improvement work from across the world, the NHS as a whole, and specifically in child health. The papers in the Equipped series that follows are full of examples, methods and tools that cut across these six domains, and which we hope will inspire and support readers to initiate projects that improve care for their patients.

Effectiveness-Simply put, effectiveness examines whether there is evidence that a treatment works. For example, McGlynn and colleagues at RAND in the USA demonstrated that only $54 \%$ of the care delivered in US hospitals met guidance that was agreed by expert consensus as being best practice. In other words, almost half of patients were not receiving the care that was known to be most effective. ${ }^{5}$ A similar study focusing on care received by children in the outpatient setting showed fewer than half of children received best practice care. ${ }^{6}$ In addition, widespread variation was found across England in a recent study on prescription patterns of 13 medications approved for national use by the National Institute for Health and Care Excellence. ${ }^{7}$ This variation was attributed to differences in diagnostic criteria, clinical uncertainty, local challenges in funding, different approaches to commissioning and variation in methods of data collection. This patchy approach to delivering effective care offers significant opportunities for improvement of care for patients worldwide.

Patient centred-this domain focuses on putting patients at the centre of healthcare. In addition to patients being the central figure within a clinical consultation, this is about empowering children, young people and their families to co-produce innovations and solutions in the design of clinical services and pathways. It is an area that will be described in more detail later on in the series.

A common feature of the hospitals in the USA that have won the 'Baldrige Award' (a Presidential Medal for high standards of care $)^{8}$ is a practice of starting each executive meeting with a patient story. Organisations such as Mercy Health System in Wisconsin have demonstrated that this simple action can focus attention on why hard decisions are made and the real reason that hospitals need to improve: the patients. Many hospitals in the NHS have adopted the 'Dr Foster Intelligent Board' approach' ${ }^{9}$ to weaving 
patient experience into the fabric of a hospital executive board's strategic functions. The development of a set of resources to guide clinicians on better communication with adolescent patients, derived from focus groups involving professionals and young people, shows how this approach, when coupled with innovative technology, can be used to improve care for children and young people. ${ }^{10}$

Timeliness - the past decade in the UK has seen a big focus on timeliness of care-the $4 \mathrm{~h}$ wait in emergency departments, the 2 week cancer wait and the 18 week wait to start consultant-led treatment. It is common to hear these discussed dismissively as 'political' targets. However, there is good evidence that they are a proxy measure for quality-for example, in Canada it has been shown that timeliness is an excellent proxy measure for system efficiency. Compared with an efficient emergency department in which overall waiting times are low, longer wait times (with inefficient processes) are associated with worse overall patient outcomes (as measured in admission and mortality rates)-irrespective of whether an individual patient is seen quickly. ${ }^{11}$ Within the NHS, a newly developed initiative is attempting to identify patientdoctor interactions that could be managed in different innovative ways-for example, Skype consultations rather than face-to-face meetings, thereby providing care when needed rather than care when certain services are open. ${ }^{12}$ Within paediatrics in the UK, recent work by NHS Improvement (a not-for-profit organisation with expertise in clinical patient pathway redesign) in paediatric audiology has identified how to improve the care given to children born with complex audiological problems. Recognising recent medical advances, healthcare organisations are redesigning their care pathways to ensure the delivery of more timely care where the patient sees the right clinician at the right time. ${ }^{13}$

Safety -one of the areas of quality that has received huge attention is that of patient safety, perhaps because it speaks directly to the underlying Hippocratic ideals of medicine: 'first do no harm'. An early and groundbreaking study that focused on harm was the Harvard Malpractice Study. This series of papers identified the levels of iatrogenic harm in patients through a retrospective case note approach. It was from this work that the Institute of Medicine was able to develop a figure of 44000-98 000 deaths from patient safety-related incidents in the USA each year. ${ }^{14-16}$ While never formally repeated in the UK to the same extent, work by Vincent et $a l^{17}$ suggests the level of harm in the NHS is comparable. One of the biggest challenges for paediatricians is making sure that the care children and young people receive is as safe as possible. The safe prescription, dispensing and administration of medications are important challenges for everyone involved in healthcare. Much of the effort to make this area safer has focused around technology, and in particular the introduction of computer-assisted prescribing systems. ${ }^{18-20}$ This is an area that is explored in more detail later on in the Equipped series.

Efficiency-describes the need to avoid waste. While the current descriptions in the literature often seek to reframe quality as 'value', it can be argued that the original definition of quality from the Institute of Medicine brings in the concepts of value through this domain of 'efficiency'. ${ }^{21}$ Waste may relate to any resource, time or money, misuse of which negatively impinges on clinical care and satisfaction. It can also include preventative approaches. In a time of global austerity, international health, which is so dependent on third sector input, is increasingly focused on efficiency rather than expansion, seeking to maximise impact from interventions known to be effective. Within the UK, an example of improving efficiency is the 'best practice tariffs'-for example, for the management of cataract. By creating a single price for a surgical procedure, organisations were forced to streamline processes. Where previously organisations were paid for every patient 'touch' (ie, each visit, investigation or operation), the bundled tariff meant a single price is paid for the complete intervention, provided all of the specified outcomes are met. This approach reduces repetitive, and often unnecessary, preoperative assessments that serve only to maximise the provider's financial benefit, and instead moves the focus on to achieving the best outcomes. This approach also has the simultaneous benefit of reducing unnecessary time spent in hospital by patients.

Examples of low-cost measures designed to improve paediatric care can be seen in the recent efforts in the UK to encourage pregnant women to have the pertussis vaccine in response to levels of disease in the community meeting epidemic levels, ${ }^{22}$ and in the recent introduction of a paediatric diabetes best practice tariff in England.

Equity - for many, the provision of a national healthcare system, free at the point of delivery, is in itself a measure of high quality, because it underscores the domain of equity, which is such a marked failing of many healthcare systems worldwide. However, recent work would suggest this alone is insufficient. Wolfe et al's ${ }^{23}$ important study demonstrates significant variation in death rates in children and young people across Europe, highlighting that outcomes in the UK are relatively poor compared with other countries in Northern and Western Europe. Within the NHS, stark variation in indicators of quality and outcomes in healthcare for children can be seen. ${ }^{24}$ While inequity in health outcomes is quite rightly a policy priority, it is complex and multifactorial. Variation in healthcare activity exposes issues with underprovision and overuse of healthcare, which are often more amenable to rapid change. ${ }^{25}$ The recent government 
response-Improving Children and Young People's Health Outcomes: A System Wide Response ${ }^{26}$ is an attempt to identify a policy solution to address both national and international variations in child health.

\section{GETTING STARTED WITH QUALITY IMPROVEMENT}

Quality improvement can be undertaken using a wide range of different tools and techniques, many of which are explained in the upcoming papers within this Equipped series. There are also excellent webbased resources available to support further learning in this area http://www.ihi.org/Pages/default.aspx. Fundamentally though, quality improvement is underpinned by the rapid audit cycle (see figure 2).

If you want to improve something the first step is identifying the area for improvement ("What needs to improve?'). Next, the indicators of success must be chosen ('What does success look like?'), and ideally, countermeasures to ensure that the change is not having a deleterious effect ('Might the change have side effects?'). Baseline measurement can then occur. Once this has happened, an intervention must be enacted and further measurement takes place ('What is the impact of the change?'). Examining the data afterwards allows honing of the intervention for future iterations ('Is there anything that could be adjusted to make the change more effective?'). After a series of small adjustments, it is hoped that improvement occurs. Fundamental to the success of any improvement is understanding the environment or context in which the intervention will occur. Key to achieving change is collaboration between clinicians, patients and families.

This approach to improvement is commonly characterised as a 'plan-do-study-act' cycle and features
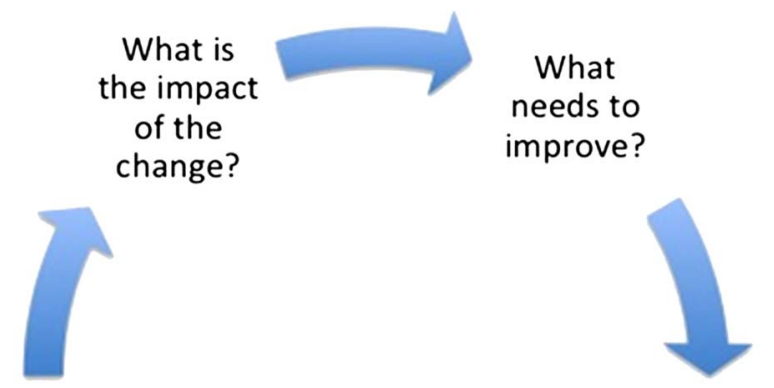

CHANGE

What does success look like?

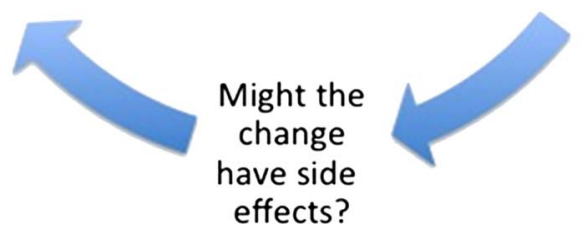

Figure 2 Driving change with the rapid audit cycle. strongly throughout the upcoming Equipped series (see figure 3). It is worth thinking about the last time you tried to improve or change anything to see if you can recognise each of the four stages in what you did. Did you manage to implement your change? Were any of the stages difficult to achieve?

Historically quality improvement has not been taught in busy undergraduate curricula, but that is slowly changing. ${ }^{27}$ Similarly, postgraduate medical education has begun to move away from expecting trainees to participate in mandatory audit towards an emphasis on gaining experience through leading bottom-up quality improvement initiatives. For the ideas and innovations of junior members of the team to be successfully implemented, they need to feel that they have permission and support to undertake this work. In departments where this is most successful, senior colleagues and trainers supervise and support trainees in their work and create an environment where they feel they can safely experiment and learn. In addition to this local support, it is important that this work is valued and the implications understood by the organisation in which the work is taking place. A number of national and international bodies, including the $\mathrm{RCPCH}$, have begun to develop a range of different activities to support learning and development in quality improvement.

\section{WHY IS QUALITY IMPROVEMENT IMPORTANT FOR HEALTHCARE?}

Quality improvement is about unceasingly seeking excellence throughout the patient journey. Beginning to see the healthcare world as one where change is possible is challenging. Few who start learning and practising quality improvement regret their changed mindset. For many clinicians rooted in a science and measurement paradigm, it is access to hard data showing measurable change in traditional outcome markers such as mortality and morbidity that makes quality improvement so compelling. For instance, in an English study replicating findings from the USA, adult intensive care units in England using a central line bundle- a set of measures to reduce infectionhave shown a mean rate decrease from 3.7 to 1.48 central venous catheter blood stream infections per 1000 central venous catheter patient days. ${ }^{28}$

For others, motivation to journey along the path of quality improvement is found through stories, like the one from Jönköping. The maternity ward here is immaculate, with each delivery room opening onto a courtyard garden and equipped with a birthing pool. But it is not the physical layout that sticks in visitors' minds. It is the low cost but priceless celebration of new life. After delivery, each mother receives a glass of 'hospital champagne' (actually fizzy apple juice) in a plastic champagne flute, served with a cocktail stick adorned with a Swedish flag and delivered to the mother on a faux silver tray. For the mother, it is an 


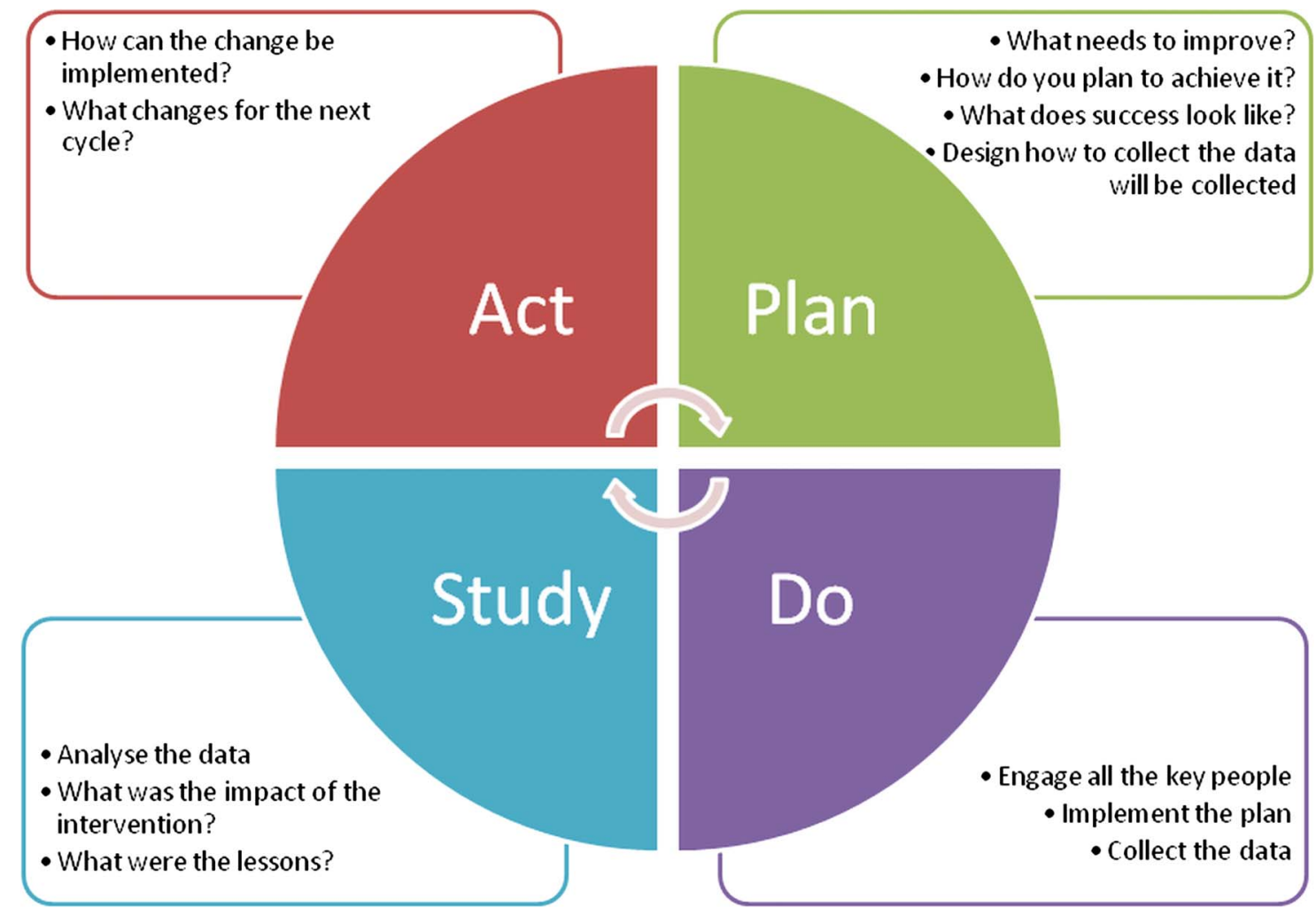

Figure 3 The plan-do-study-act cycle.

opportunity to celebrate and reflect. For the organisation, it is a way of acknowledging their part in this extraordinary event in the lives of their patients. For the observer, it is a powerful reminder of what is possible, if a culture of quality improvement is truly embedded within the healthcare environment.

Quality improvement is about changing behaviours, approaches and systems to maximise the quality of care that patients receive. Crucially, more than just a rigorous focus on changing numbers and values, quality improvement seeks to change the culture within which healthcare is delivered. Quality improvement is about clinicians believing, as those at Jönköping do, that their roles include an unrelenting focus on making things better. It is not enough to just do the day job. It is about focusing on the patient and making healthcare meet their needs.

Paediatricians, it seems, understand this instinctively. Many of the international leaders in quality improvement have a clinical background in paediatrics:

- Don Berwick-Former Chief Executive Officer of the Institute of Healthcare Improvement (IHI)

- Paul Batalden-Senior Fellow of IHI and one of the leading academic thinkers on quality improvement

- Amitai Ziv-Paediatrician, ex-Air Force pilot and early protagonist of simulation as a means to improve the delivery of care

In addition, many of the trainee-led quality improvement initiatives in the UK have paediatricians at their heart. ${ }^{29}$ Perhaps it is something about the strong patient focus of our clinical work or the tenacious desire to improve things for patients, but paediatricians have an outstanding track record of leading quality improvement work.

The current situation in healthcare means focusing on quality improvement is imperative. With the publication of the Francis report ${ }^{30}$ in the UK, it is clear that fundamental quality elements such as patient-centredness have become lost within the unrelenting focus on achieving key performance measures. Similarly, the need to control the escalating costs of healthcare across the world will not happen with solely a top-down approach.

This unrelenting approach to continuous improvement needs to sit with us as paediatricians, and with our colleagues, on the front line. Each of us needs to see it as part of our role to think about how to change services to make them function more effectively. We must learn how to adapt and change our practice in order to keep pace with evolving expectations and patient needs. Above all, we need to think about every aspect of what we do and embrace how we can do it better.

Acknowledgements We thank Ian Wacogne for his support and vision in conceiving and designing the Equipped series and in reviewing and critiquing this article.

Contributors CL, CRLHC and REK were all involved in the planning and design of the article, the production of revised drafts and final approval of the version to be published.

Competing interests Dr Bob Klaber is the RCPCH Officer for Educational Provision. 
Provenance and peer review Commissioned; internally peer reviewed.

\section{REFERENCES}

1 Institute of Medicine. Crossing the quality chasm: a new health system for the 21st century. Washington, DC: National Academy Press, 2001.

$2 \mathrm{RCPCH}$. Facing the future: standards for paediatric services. London: RCPCH, 2010.

$3 \mathrm{RCPCH}$. Back to facing the future: an audit of standards for acute paediatric care. London: RCPCH, 2013.

4 Runnacles J, Moult B, Lachman P. Developing future clinical leaders for quality improvement: experience from a London children's hospital. BMJ Qual Saf Published Online First: 9 July 2013 doi:10.1136/bmjqs-2012-001718.

5 McGlynn EA, Asch SM, Adams J, et al. The quality of health care delivered to adults in the United States. N Engl J Med 2003;348:2635-45.

6 Mangione-Smith R, DeCristofaro AH, Setodji CM, et al. The quality of ambulatory care delivered to children in the United States. N Engl J Med 2007;357:1515-23.

7 Stephens P. Bridging the gap: why some people are not offered the medicines that NICE recommends [Internet]. London, UK: IMS Health Inc, 2012. http://www.imshealth.com/ims/Global/Content/ Insights/IMS20Institute20for20Healthcare20Informatics/NICE\% 20report/IMS_Health_Bridging_the_Gap_2012.pdf (accessed 31 May 2013).

8 US Department of Commerce N. Baldrige Homepage [Internet]. http://www.nist.gov/baldrige/ (accessed 31 May 2013).

9 The Intelligent Board: Patient Experience [Internet]. Dr Foster Intelligence. 2010. http://drfosterintelligence.co.uk/wp-content/ uploads/2011/06/Intelligent-Board-2010.pdf (accessed 31 May 2013).

10 Hamilton S. The Talk Lab [Internet]. London, UK. http:// talklab.nhs.uk/menu.html (accessed 31 May 2013).

11 Guttmann A, Schull MJ, Vermeulen MJ, et al. Association between waiting times and short term mortality and hospital admission after departure from emergency department: population based cohort study from Ontario, Canada. BMJ 2011;342:d2983.

12 Productive Primary Care Ltd. Doctor First [Internet]. http:// www.productiveprimarycare.co.uk/doctor-first.aspx (accessed 31 May 2013).

13 NHS Improvement. Children's hearing services [Internet]. http:// www.improvement.nhs.uk/audiology/documents/audiology_case_ studies/warwickshire.pdf (accessed 31 May 2013).

14 Brennan TA, Leape LL, Laird NM, et al. Incidence of adverse events and negligence in hospitalized patients. Results of the Harvard Medical Practice Study I. N Engl J Med 1991;324:370-6.
15 Brennan TA, Leape LL, Laird NM, et al. Incidence of adverse events and negligence in hospitalized patients: results of the Harvard Medical Practice Study I. 1991. Qual Saf Health Care 2004;13:145-51.

16 Kohn L, Corrigan J, Donaldson M. To err is human: building a safer health system [Internet]. Washington, DC: National Academy Press, 2000. http://www.nap.edu/openbook.php? isbn $=0309068371$ (accessed 31 May 2013).

17 Vincent C, Neale G, Woloshynowych M. Adverse events in British hospitals: preliminary retrospective record review. BMJ 2001;322:517-19.

18 Kaushal R, Bates DW, Landrigan C, et al. Medication errors and adverse drug events in pediatric inpatients. Jama J Am Med Assoc 2001;285:2114-20.

19 Van Rosse F, Maat B, Rademaker CMA, et al. The effect of computerized physician order entry on medication prescription errors and clinical outcome in pediatric and intensive care: a systematic review. Pediatrics 2009;123:1184-90.

20 Abramson EL, Kaushal R. Computerized provider order entry and patient safety. Pediatr Clin North Am 2012;59: 1247-55.

21 Porter ME. What is value in health care? N Engl J Med 2010;363:2477-81.

22 Choices NHS. Whooping cough vaccination in pregnancyPregnancy and baby guide-NHS Choices [Internet]. 2013. http:// www.nhs.uk/Conditions/pregnancy-and-baby/Pages/whoopingcough-vaccination-pregnant.aspx (accessed 31 May 2013).

23 Wolfe I, Thompson M, Gill P, et al. Health services for children in western Europe. Lancet 2013;381:1224-34.

24 Cheung CRLH, Gray JAM. Unwarranted variation in health care for children and young people. Arch Dis Child 2013;98: 60-5.

25 NHS atlas of variation: problems of the gastro-intestinal system [Internet]. London, UK: Crown Copyright, 2011. http://www. rightcare.nhs.uk/atlas/downloads/Gastrointestinal_AoV_2011. pdf (accessed 31 May 2013).

26 Department of Health. Improving children and young people's health outcomes: a system wide response. London, UK: Crown Copyright, 2013.

27 Walton M, Woodward H, Van Staalduinen S, et al. Republished paper: the WHO patient safety curriculum guide for medical schools. Postgrad Med J 2011;87:317-21.

28 Bion J, Richardson A, Hibbert P, et al. 'Matching Michigan': a 2-year stepped interventional programme to minimise central venous catheter-blood stream infections in intensive care units in England. BMJ Qual Saf 2013;22:110-23.

29 NHS Change Day [Internet]. http://www.changemodel.nhs.uk/ $\mathrm{pg} /$ groups $/ 33183 / \mathrm{NHS}+$ Change + Day $/ 27$ ? community $=\mathrm{NHS}$ +Change+Day (accessed 31 May 2013).

30 Francis R. Report of the mid Staffordshire NHS Foundation Trust public inquiry. London: The Stationary Office, 2013. 